This manuscript is accepted by Ap\&SS

\title{
Matter Infall in Collapsing Molecular Cloud Cores with an Axial Magnetic Field
}

\author{
Mohsen Nejad-Asghar \\ Department of Physics, University of Mazandaran, Babolsar, Iran \\ nejadasghar@umz.ac.ir
}

\begin{abstract}
The magnetic fields affect collapse of molecular cloud cores. Here, we consider a collapsing core with an axial magnetic field and investigate its effect on infall of matter and formation of accretion disk. For this purpose, the equations of motion of ions and neutral infalling particles are numerically solved to obtain the streamlines of trajectories. The results show that in non-steady state of ionization and ion-neutral coupling, which is not unexpected in the case of infall, the radius of accretion disk will be larger as a consequence of axial magnetic field.
\end{abstract}

Subject headings: star: formation - ISM: clouds - ISM: evolution - magnetic fields

\section{Introduction}

A lot of observational information is now known about the structure of dense cores as progenitors of stars within the molecular clouds (e.g., Evans 2010). Theoretically, we expect that most of these cores will collapse into protostars, but the details of evolutions are less evident. Clearly, the evolution heavily depends upon the effects of local density, pressure, rotation, magnetic fields, the presence or absence of nearby stars and protostars, and upon the other physical phenomena. The most commonly used picture of low-mass star formation is inside-out collapse (Shu 1977) in which it begins at the center of a singular isothermal sphere and an expanding wave of infalling matter propagates outward with the speed of sound. Up to now, different models for core collapse and formation of protostars have been presented, in each the effects of some physical phenomena investigated (e.g., McKee and Ostriker 2007). 
The velocity maps of molecular cloud cores, which are built by using the velocity measurements along the line of sight, show a small velocity gradient across each core. Measuring this global velocity gradient, with assumption that core has a uniform rotation and follow a rigid-body rotation law, can be used to deduce the core angular velocity (e.g., Goodman et al. 1993). The large size of initial collapsing core implies that even the modest initial rotational velocities will cause the infalling matters to land first on a rotationally supported disk rather than a pressure-supported proto-star (e.g., Hartmann 2009, Nejad-Asghar 2011). In the simplest analysis of rotating collapse, we assume that pressure forces are negligible and so the problem can be analyzed by using ballistic trajectories. The results for collapse of a spherically symmetric cloud in uniform (solid-body) rotation were initially worked out by Ulrich (1976), with subsequent extension to disk formation by Cassen and Moosman (1981), and to collapsing singular isothermal sphere by Terebey, Shu and Cassen (1984). Mendoza, Tejeda and Nagel (2009, hereafter MTN) have recently generalized this idea by construction a steady analytic accretion flow model for a finite rotating gas cloud. They show that the streamlines and density profiles deviate considerably from ones calculated by Ulrich, and for all relevant astrophysical situations, the assumption of a finite cloud radius needs to be used.

The observations indicate that magnetic energy in the molecular clouds is comparable to the gravitational energy (e.g., Crutcher 1999). Moreover, the magnetic fields are also theoretically believed to play an important role in gravitational collapse of the molecular cloud cores. They provide pressure support against the gravity and carries away angular momentum prior to and during the collapse of cores to form accretion disks, jets and protostars (e.g., Machida 2010). Although, in a molecular cloud core, the spatial configuration of magnetic field lines is not simple (e.g., Whittet 2005), but polarimetry observations of young stellar objects suggest that circumstellar thin disks around the young stars have approximately aligned perpendicular to the magnetic fields (e.g., Pereyra et al. 2009). Here, we consider a simple initial configuration in which the magnetic fields are assumed to be parallel with rotational axis of core. Since the molecular cloud cores are lightly-ionized, the ambipolar diffusion in which the magnetic field is frozen into the charged species and drifts along with them through neutrals, is an important mechanism (e.g., Adams 2009). Thus, we consider the effect of magnetic fields directly on charged particles, while the neutral species feel them indirectly via the collisions with ions.

In this way, as an extension to the work of MTN, we investigate the effect of axial magnetic field on streamlines of infalling ions and neutral particles. For this purpose, formulation of problem and equations of motion are given in section 2. In section 3, trajectories of infalling ions and neutral particles are investigated and effect of the magnetic fields on the accretion disk radius is studied. Finally, section 4 is allocated to summary and conclusions. 


\section{Formulation of problem}

The molecular cloud cores are lightly-ionized gases with small fraction of ions with density $n_{i}$ and electrons with density $n_{e} \approx n_{i}$. If the volumetric rate of electron-ions recombination took place in gas phase, it would be proportional to $n_{e} n_{i} \propto n_{i}^{2}$. In a simple steady state approximation, the volumetric rate of electron-ions recombination $\propto n_{i}^{2}$ would be equal to the volumetric rate of neutrals ionization via cosmic rays $\propto n_{n}$. Although, the actual ionization balance in the molecular clouds is more complicated, Elmegreen (1979) and Umebayashi and Nakano (1980) showed that the relation $n_{i} \propto n_{n}^{1 / 2}$ is a good approximation. Here, we suppose for fiducial purpose that $\rho_{i}=\tilde{\epsilon} \epsilon \rho_{n}^{1 / 2}$, where $\epsilon=9.5 \times 10^{-15} \mathrm{~m}^{-3 / 2} \mathrm{~kg}^{1 / 2}$ and $\tilde{\epsilon}$ is a dimensionless free parameter which represents the deviations from calculations in the steady state approximation.

In the magnetized cloud cores, the mean velocity $\mathbf{v}_{n}$ of neutrals will not generally equal to the mean velocity $\mathbf{v}_{i}$ of ions and $\mathbf{v}_{e}$ of electrons. Although, the positive ions and electrons feel the magnetic forces in opposite direction, but the induced electric field will generally cause they move in ambipolar motion, so that in the time-scales that are considered here, their mean velocities are approximately the same $\left(\mathbf{v}_{e} \approx \mathbf{v}_{i}\right)$. Since electrons carry much less momentum than ions, we neglect the dynamic of electrons. In this way, resistance of relative drift between ions and neutrals will be a drag acceleration arises from mutual collisions between them. The drag acceleration exerted on neutrals by ions is $\tilde{\gamma} \gamma \rho_{i}\left(\mathbf{v}_{i}-\mathbf{v}_{n}\right)$; the drag acceleration exerted on ions by neutrals is $-\tilde{\gamma} \gamma \rho_{n}\left(\mathbf{v}_{i}-\mathbf{v}_{n}\right)$, where $\gamma=3.5 \times 10^{10} \mathrm{~m}^{3} \mathrm{~kg}^{-1} \mathrm{~s}^{-1}$ (Draine, Roberge and Dalgarno 1983) is the drag coefficient and $\tilde{\gamma}$ is a dimensionless free parameter which represents the deviations from calculations in the steady state approximation.

The equations of motion for ions and neutral particles, in gravitational field of a central mass, are respectively as follows

$$
\begin{gathered}
\ddot{\mathbf{r}}_{i}=-\frac{G M}{r_{i}^{3}} \mathbf{r}_{i}+\frac{q_{i}}{m_{i}} \dot{\mathbf{r}}_{i} \times \mathbf{B}-\tilde{\gamma} \gamma \rho_{n}\left(\dot{\mathbf{r}}_{i}-\dot{\mathbf{r}}_{n}\right), \\
\ddot{\mathbf{r}}_{n}=-\frac{G M}{r_{n}^{3}} \mathbf{r}_{n}+\tilde{\epsilon} \tilde{\gamma} \epsilon \gamma \rho_{n}^{1 / 2}\left(\dot{\mathbf{r}}_{i}-\dot{\mathbf{r}}_{n}\right),
\end{gathered}
$$

where $M$ is the proto-stellar mass at the origin, and $q_{i}=N_{1} e$ and $m_{i}=N_{2} m_{p}$ are ion charge and mass, respectively, as an integer multiple of the electron charge $e$ and the proton mass $m_{p}$. Here, we assume that $N_{2} \approx 2 N_{1}$.

We measure the length and time in units of $0.01 \mathrm{pc}$ and $10^{5} \mathrm{yr}$, respectively, so that velocity unit is $0.1 \mathrm{~km} \mathrm{~s}^{-1}$. Mass unit is appointed to $1 M_{\odot}$, thus the gravitational constant is $G=45$. In this manner, we have $\gamma=7.8 \times 10^{9}$ and $\epsilon=3.5 \times 10^{-8}$. Finally, the unit 
of magnetic field is chosen equal to $10 \mathrm{nT}=100 \mu G$. Using these units, the equations of motion (11) and (2) can be rewritten in a dimensionless form as follows

$$
\begin{gathered}
\ddot{\mathbf{r}}_{i}=-45 \frac{M}{r_{i}^{3}} \mathbf{r}_{i}+0.5 \tilde{B} \dot{\mathbf{r}}_{i} \times \hat{\mathbf{B}}-3.9 \times 10^{5} \tilde{\gamma} \tilde{n}_{n}\left(\dot{\mathbf{r}}_{i}-\dot{\mathbf{r}}_{n}\right), \\
\ddot{\mathbf{r}}_{n}=-45 \frac{M}{r_{n}^{3}} \mathbf{r}_{n}+1.9 \tilde{\epsilon} \tilde{\gamma} \tilde{n}_{n}^{1 / 2}\left(\dot{\mathbf{r}}_{i}-\dot{\mathbf{r}}_{n}\right),
\end{gathered}
$$

where $\hat{\mathbf{B}}$ is a unit vector in the direction of magnetic field, and $\tilde{n}_{n} \equiv n_{n} /\left(10^{10} \mathrm{~m}^{-3}\right)$ and $\tilde{B}$ are two dimensionless free parameters.

Choosing the axial magnetic field $\hat{\mathbf{B}}=\hat{k}$, the equations of motion in Cartesian coordinate are

$$
\begin{gathered}
\ddot{x}_{i}=-45 M \sin \theta_{i} \cos \phi_{i} \frac{1}{r_{i}^{2}}+0.5 \tilde{B} \dot{y}_{i}-3.9 \times 10^{5} \tilde{\gamma} \tilde{n}_{n}\left(\dot{x}_{i}-\dot{x}_{n}\right), \\
\ddot{y}_{i}=-45 M \sin \theta_{i} \cos \phi_{i} \frac{1}{r_{i}^{2}}-0.5 \tilde{B} \dot{x}_{i}-3.9 \times 10^{5} \tilde{\gamma} \tilde{n}_{n}\left(\dot{y}_{i}-\dot{y}_{n}\right), \\
\ddot{z}_{i}=-45 M \sin \theta_{i} \cos \phi_{i} \frac{1}{r_{i}^{2}}-3.9 \times 10^{5} \tilde{\gamma} \tilde{n}_{n}\left(\dot{z}_{i}-\dot{z}_{n}\right), \\
\ddot{x}_{n}=-45 M \sin \theta_{n} \cos \phi_{n} \frac{1}{r_{n}^{2}}+1.9 \tilde{\epsilon} \tilde{\gamma} \tilde{n}_{n}^{1 / 2}\left(\dot{x}_{i}-\dot{x}_{n}\right), \\
\ddot{y}_{n}=-45 M \sin \theta_{n} \cos \phi_{n} \frac{1}{r_{n}^{2}}+1.9 \tilde{\epsilon} \tilde{\gamma} \tilde{n}_{n}^{1 / 2}\left(\dot{y}_{i}-\dot{y}_{n}\right),
\end{gathered}
$$

where $\theta_{i}, \theta_{n}, \phi_{i}$, and $\phi_{n}$ are the polar and azimuthal angles of ions and neutral particles, respectively, and $r_{i}=\sqrt{x_{i}^{2}+y_{i}^{2}+z_{i}^{2}}$ and $r_{n}=\sqrt{x_{n}^{2}+y_{n}^{2}+z_{n}^{2}}$ are their radial distances. There are four free parameters $\tilde{B}, \tilde{\epsilon}, \tilde{\gamma}$, and $\tilde{n}_{n}$ which represent the strength of magnetic field, ionization fraction, drag coefficient, and neutral density, respectively.

\section{Trajectories of infalling matters}

As mentioned before, there are many observations that show velocity gradients in the maps of molecular cloud cores. These velocity gradients can be used to deduce the rotation of cores, but evaluation of their angular velocity $\Omega_{0}$ is considerably complicated because the velocity fields usually exhibit complex supersonic motions. Here, we choose the fiducial value of $\Omega_{0}=3 \times 10^{-13} \mathrm{rad} \mathrm{s}^{-1}$, which in the time unit $10^{5} \mathrm{yr}$ is $\Omega_{0} \approx 1$. The effects of initial angular momentum in core collapse and formation of accretion disks are now approximately 
well known (e.g., Hartmann 2009). Here, we turn our attention to the effects of magnetic fields and drag force on infalling matters. If we assume that $\tilde{\gamma}=0$ and $\tilde{B}=0$, equations (8) -(17) lead to the trajectories which are contained on a plane and given by a conic section (i.e., the results obtained by MTN).

Choosing the initial conditions

$$
\begin{aligned}
\mathbf{r}_{n}=\mathbf{r}_{i}= & r_{0} \hat{r} \\
= & r_{0} \sin \theta_{0} \cos \phi_{0} \hat{i}+r_{0} \sin \theta_{0} \sin \phi_{0} \hat{j}+r_{0} \cos \theta_{0} \hat{k}, \\
\mathbf{v}_{n}=\mathbf{v}_{i}=-v_{0 r} \hat{r}+v_{0 \phi} \hat{\phi}= & -\left(v_{0 r} \cos \phi_{0}+r_{0} \sin \phi_{0}\right) \sin \theta_{0} \hat{i} \\
& -\left(v_{0 r} \sin \phi_{0}-r_{0} \cos \phi_{0}\right) \sin \theta_{0} \hat{j}-v_{0 r} \cos \theta_{0} \hat{k},
\end{aligned}
$$

for position and velocity of infalling particles, the equations of motion (5)-(10) can be solved by numerical methods such as Runge-Kutta. Approximately, all cores in the maps of molecular clouds seem apparently to be elongated rather than spherical (e.g., Curtis and Richer 2010). Since determining the exact three-dimensional shape of a core from apparent observations of the-plane-of-sky is impossible, statistical techniques have to be applied; as the results, some works indicate a preference for prolate cores (e.g., Gammie et al. 2003, Li et al. 2004), and some others favour oblate shapes (e.g., Curry 2002, Jones and Basu 2002). Theoretically, the prolate elongation of the cores may be inferred as a remnant of their origin in filaments and turbulent flows (e.g., Hartmann 2002), while the oblate shape of collapsing cores may be justified from the effects of magnetic fields and rotational motion (e.g., NejadAsghar 2010). The streamlines of infalling particles, in absence of magnetic field $(\tilde{B}=0$, $\tilde{\gamma}=0)$ and initial radial velocity $\left(v_{0 r}=0\right)$, for initial spherical core $\left(r_{0}=1\right)$, oblate core $\left(r_{0}=1 / \sqrt{\sin ^{2} \theta_{0}+4 \cos ^{2} \theta_{0}}\right)$, and prolate core $\left(r_{0}=1 / \sqrt{4 \sin ^{2} \theta_{0}+\cos ^{2} \theta_{0}}\right)$ are shown in Fig,1.

The numerical plots of accretion disk radius for neutral particles by substitution $\theta_{0} \rightarrow$ $\pi / 2$, for a wide variety of initial parameters, are shown in Fig. 2. The numerical results show that the accretion disk radius is approximately proportional to the inverse of initial radial velocity $v_{0 r}$ as was given by the analytical ballistic models of MTN. Since we want to investigate the effect of magnetic fields on trajectories of infalling matters, without losing the generality of problem, we consider an initial spherical core with assumption $v_{0 r}=0$. Here we turn our attention to only one streamline with $r_{0}=1$ and $\theta_{0}=\pi / 4$. The effects of axial magnetic field on this streamline are shown in Fig 3 for three different values of free parameter $\tilde{B}$ with a fixed value of three parameters $\tilde{\gamma}, \tilde{\epsilon}$ and $\tilde{n}_{n}$ equal to $10^{-5}, 10^{5}$, and 1 , respectively. As a result, we see that the axial magnetic field causes the infalling ions land at a larger equatorial radius, and collisions of ions with neutral particles lead to the 
same behavior for infalling neutral matters. In Fig, 4, the equatorial radii of ion and neutral infalling particles (from $\theta_{0}=\pi / 4$ ) are depicted versus $\tilde{\gamma}$ and $\tilde{\epsilon}$ at a fixed value of magnetic field. We see that increasing of drag coefficient $\tilde{\gamma}$ leads to coupling of ions and neutrals so that their equatorial radius reach to each other asymptotically. The same behavior can be seen for the effect of ionization degree $\tilde{\epsilon}$ so that increasing of this parameter asymptotically causes to approximately the same equatorial radii of ions and neutral infalling particles. The third free parameter is neutral density $\tilde{n}_{n}$ which its effect is the same as $\tilde{\gamma}$ but its effect on neutral particles is less.

The density of neutral particles can be evaluated by assuming that the mass infall rate, $\dot{M}$, is steady. Assuming that the cloud at $r_{0}$ is nearly spherical, the mass flow in a flow tube spanned by $\triangle \theta_{0}$ is

$$
\triangle \dot{M}=\frac{2 \pi r^{2} \sin \theta_{0} \dot{M} \triangle \theta_{0}}{4 \pi r^{2}}=\frac{1}{2} \dot{M} \sin \theta_{0} \triangle \theta_{0} .
$$

The density at $(r, \theta)$ can be found by following the streamlines corresponding to $\theta_{0}$ and $\theta_{0}+\triangle \theta_{0}$

$$
\rho(r, \theta)=\frac{\triangle \dot{M}}{2 \pi r^{2} \sin \theta \triangle \theta\left|v_{r}\right|}=\frac{\dot{M}}{4 \pi r^{2}\left|v_{r}\right|} \frac{\sin \theta_{0}}{\sin \theta} \frac{\triangle \theta_{0}}{\triangle \theta} .
$$

In order to obtain the iso-density contours, we divide $0 \leq \theta_{0} \leq \pi / 2$ to $N$ equal segments so that $\triangle \theta_{0}=\pi / 2 N$. For two streamlines in the borders of each segment, values of $\sin \theta_{0}$, $\sin \theta$ and $v_{r}$ are evaluated in the mid points. In this way, the density at different radii and polar angles can be found from (14), and the iso-density curves can be depicted as typically are shown in Fig. 5. In this figure, the mass accretion rate is assumed to be $\dot{M}=4 \pi \times 10^{-7} \mathrm{M}_{\odot} \mathrm{yr}^{-1}$. These iso-density contours show that the axial magnetic fields cause the infalling matters land at a larger equatorial radius.

\section{Summary and conclusions}

Nowadays, we have accepted that the molecular cloud cores can be gravitationally unstable under certain conditions, and they can collapse. Rotational motion of the cores leads to land the infalling matters on an accretion disk around the proto-star. The streamlines of infalling matters for initial spherical, oblate and prolate cores are depicted in Fig!1. Obviously, the centrifugal force causes to increase the initial dimension of accretion disk in oblate cores, while it decreases the disk radius in prolate ones.

Next, we considered an axil magnetic field in the collapsing core. For investigation the effect of this magnetic field, we wrote separately the equations of motion of neutral and ion infalling particles. In general form, these equations are presented with four free parameters 
$\tilde{B}, \tilde{\epsilon}, \tilde{\gamma}$, and $\tilde{n}_{n}$ which represent the strength of magnetic field, ionization fraction, drag coefficient, and neutral density, respectively. The accretion disk radius for different values of the relevant input parameters are depicted in Fig.2. Since our goal is to study the effect of magnetic field and drag coefficient on infalling matters and disk formation, we turned our attention to only one streamline at $\theta_{0}=\pi / 4$; the results are shown in Fig. 3 .

In a steady state of molecular gas, the ions are well-coupled to neutral particles (i.e., $\tilde{\epsilon}=1, \tilde{\gamma}=1$ ) so that their dynamics are approximately the same. Fig 4 shows that increasing of drag coefficient and ionization degree lead to the same behavior for ions and neutral infalling particles. In infalling case, the frequency of collisions between ions and neutral particles eventually becomes so low that the balance cannot be maintained, thus, the values

of free parameters $\tilde{\epsilon}$ and $\tilde{\gamma}$ deviate from the steady sate. In this non-equilibrium case, ions are directly affected by magnetic field so that they land in a larger radii at equatorial plane. In the low-coupling $(\tilde{\gamma}<<1)$ and high-ionization degree $(\tilde{\epsilon}>>1)$, the dynamics of ions can affect the motions of neutral particles so that they also land in a larger radii to form the larger accretion disk as shown in Fig.3. The same behavior for increasing of accretion disk radius via the effect of magnetic field can be deduced from iso-density contours that are depicted in Fig. 5,

\section{Acknowledgments}

This work has been supported by grant of Research and Technology Deputy of University of Mazandaran.

\section{REFERENCES}

Adams F.C., 2009, RMxAC, 36, 73

Cassen P., Moosman A., 1981, Icarus, 48, 353

Crutcher R.M., 1999, ApJ, 520, 706

Curtis E.I., Richer J.S., 2010, MNRAS, 402, 603

Curry C.L., 2002, ApJ, 576, 849

Draine B.T., Roberge W.G., Dalgarno A., 1983, ApJ, 264, 485

Elmegreen B.G., 1979, ApJ, 232, 729 
Evans N.J., 2010, in Proceedings of IAU Symposium 270: Computational Star Formation, Barcelona, Spain, eds.: Alves, Elmegreen, Girart, Trimble, arXiv1007.5214

Gammie C.F., Lin Y., Stone J.M., Ostriker E.C., 2003, ApJ, 592, 203

Goodman A.A., Benson P.J., Fuller G.A., Myers P.C., 1993, ApJ, 406, 528

Hartmann L., 2002, ApJ, 578, 914

Hartmann L., 2009, Accresion Processes in Star Formation, 2ed, Cambridge University Press

Jones C.E., Basu S., 2002, ApJ, 569, 280

Li P.S., Norman M.L., MacLow M., Heitsch F., 2004, ApJ, 605, 800

Machida M.N., 2010, in Proceedings of the IAU Symposium 270: Computational Star Formation, Barcelona, Spain, eds.: Alves, Elmegreen, Girart, Trimble, arXiv1008.0929

McKee C.F., Ostriker E.C., 2007, ARA\&A, 45, 565

Mendoza S., Tejeda E., Nagel E., 2009, MNRAS, 393, 579 (MTN)

Nejad-Asghar M., 2011, AN, in press (arXiv1102.2505)

Nejad-Asghar M., 2010, RAA, 10, 1275

Pereyra A., Girart J.M., Magalhães A.M., Rodrigues C.V., de Araújo F.X., 2009, A\&A, 501, 595

Shu F.H., 1977, ApJ, 214, 488

Terebey S., Shu F.H., Cassen P., 1984, ApJ, 286, 529

Ulrich R.K., 1976, ApJ, 210, 377

Umebayashi T., Nakano T., 1980, PASJ, 32, 405

Whittet D.C.B., 2005, ASPC, 343, 321 


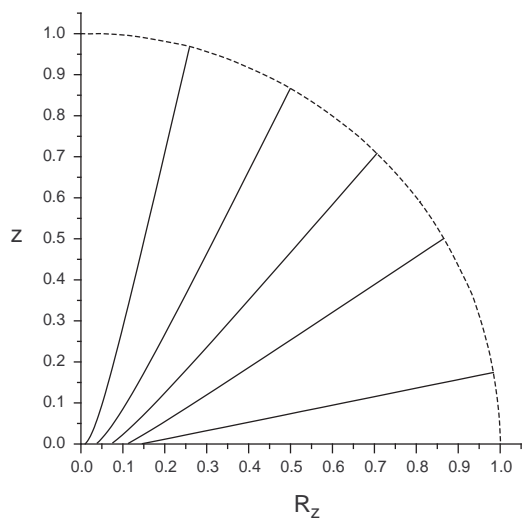

(a)

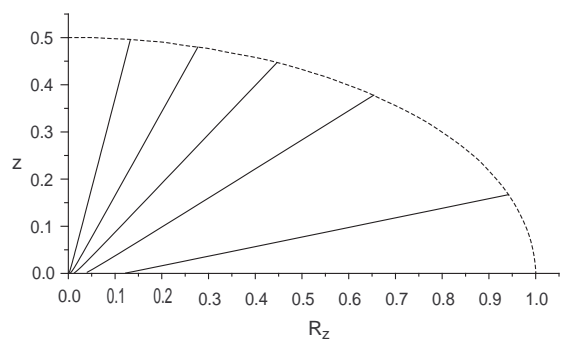

(b)

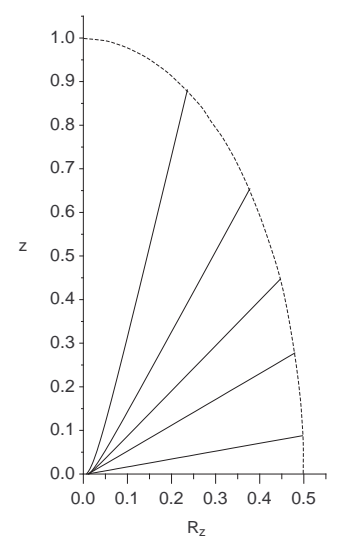

(c)

Fig. 1.- The streamlines of ions and neutral particles in absence of magnetic field and initial radial velocity $\left(\tilde{B}=0, \tilde{\gamma}=0, v_{0 r}=0\right)$, for (a) initial spherical core $\left(r_{0}=1\right)$, (b) initial oblate core $\left(r_{0}=1 / \sqrt{\sin ^{2} \theta_{0}+4 \cos ^{2} \theta_{0}}\right)$, and (c) initial prolate core $\left(r_{0}=1 / \sqrt{4 \sin ^{2} \theta_{0}+\cos ^{2} \theta_{0}}\right)$. In each figure, the curves from top to bottom have the initial azimuthal angle equal to $\theta_{0}=15,30,45,60$, and $80^{\circ}$. 

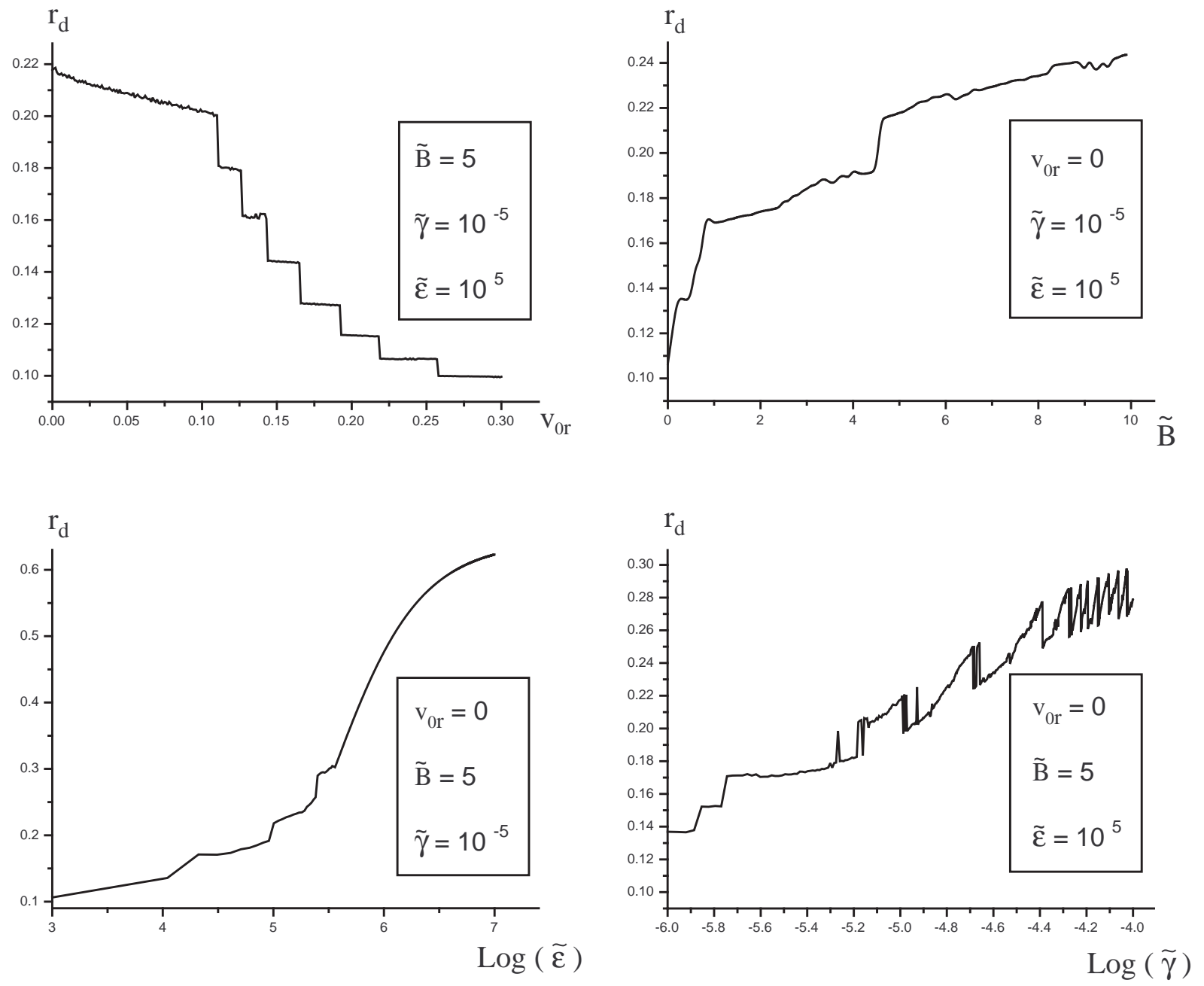

Fig. 2.- Accretion disk radius by the substitution $\theta_{0} \rightarrow \pi / 2$ for various initial free parameters with $\tilde{n}=1$. 


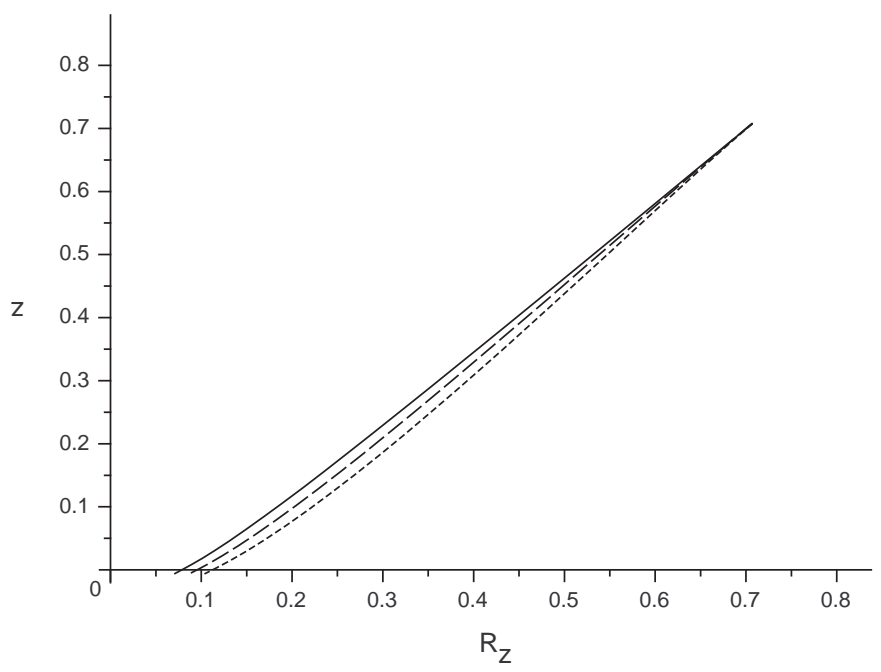

(a)

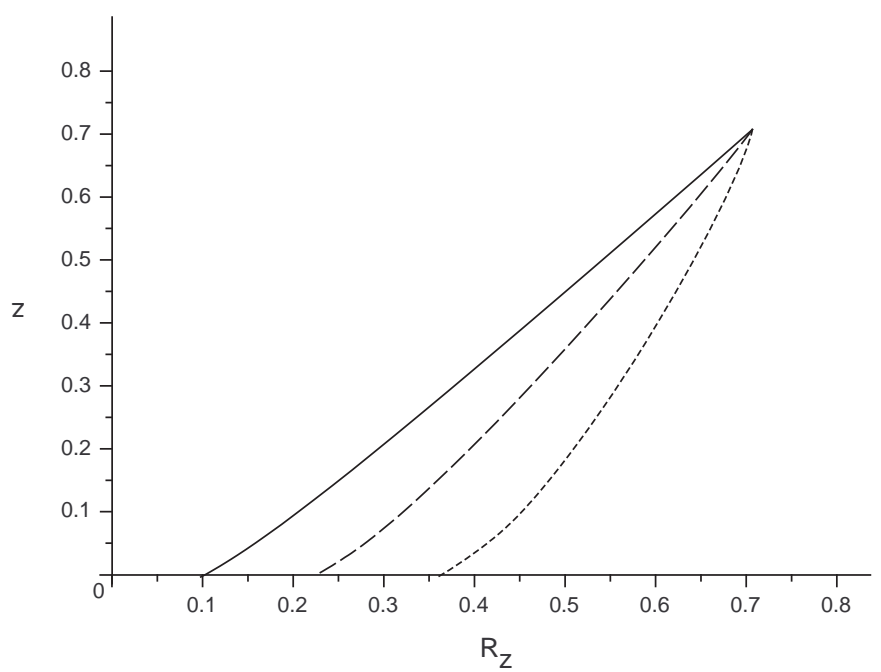

(b)

Fig. 3.- The streamlines of (a) neutral and (b) ion flows for different values of free parameter $\tilde{B}=1$ (solid), $\tilde{B}=5$ (dash), and $\tilde{B}=10$ (dot), at $\theta_{0}=\pi / 4$ with $\tilde{\gamma}=10^{-5}, \tilde{\epsilon}=10^{5}$ and $\tilde{n}_{n}=1$. 


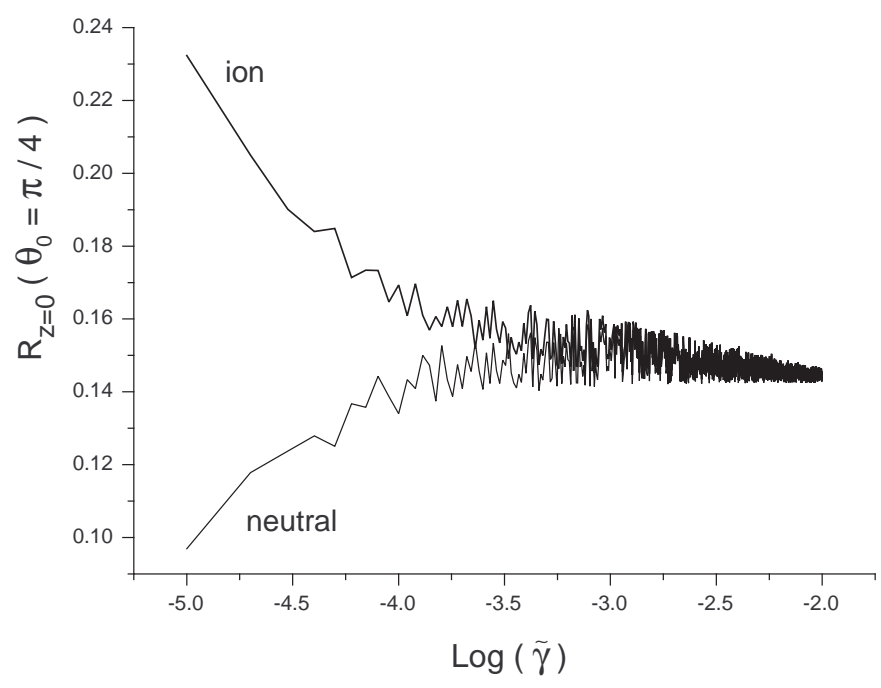

(a)

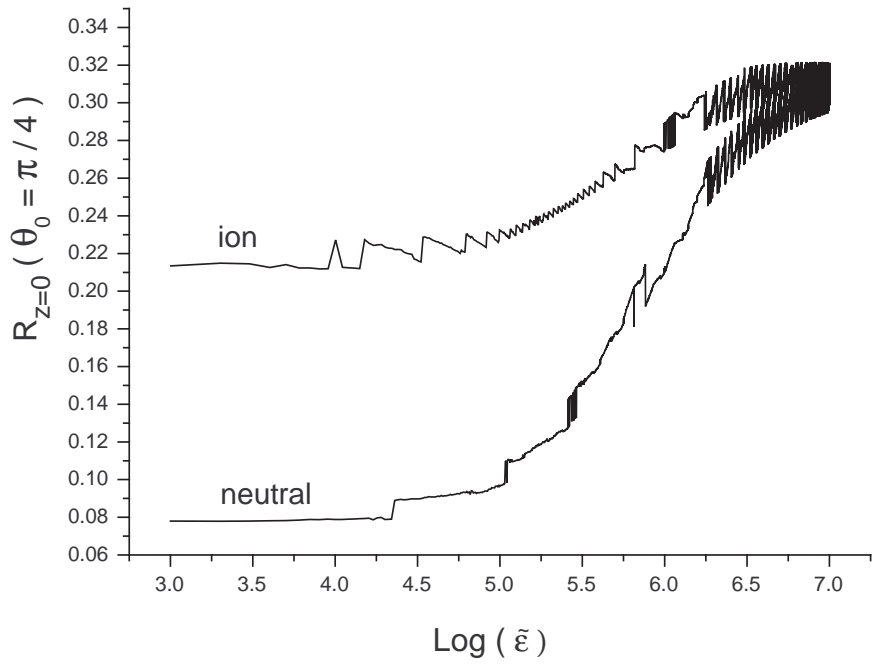

(b)

Fig. 4.- The equatorial radius of infalling ions and neutral particles which start at $\theta_{0}=\pi / 4$, versus (a) logarithm of free parameter $\tilde{\gamma}$ with $\tilde{\epsilon}=10^{5}$, and (b) logarithm of free parameter $\tilde{\epsilon}$ with $\tilde{\gamma}=10^{-5}$. In these figures the free parameters $\tilde{B}$ and $\tilde{n}_{n}$ are chosen equal to 5 and 1 , respectively. 


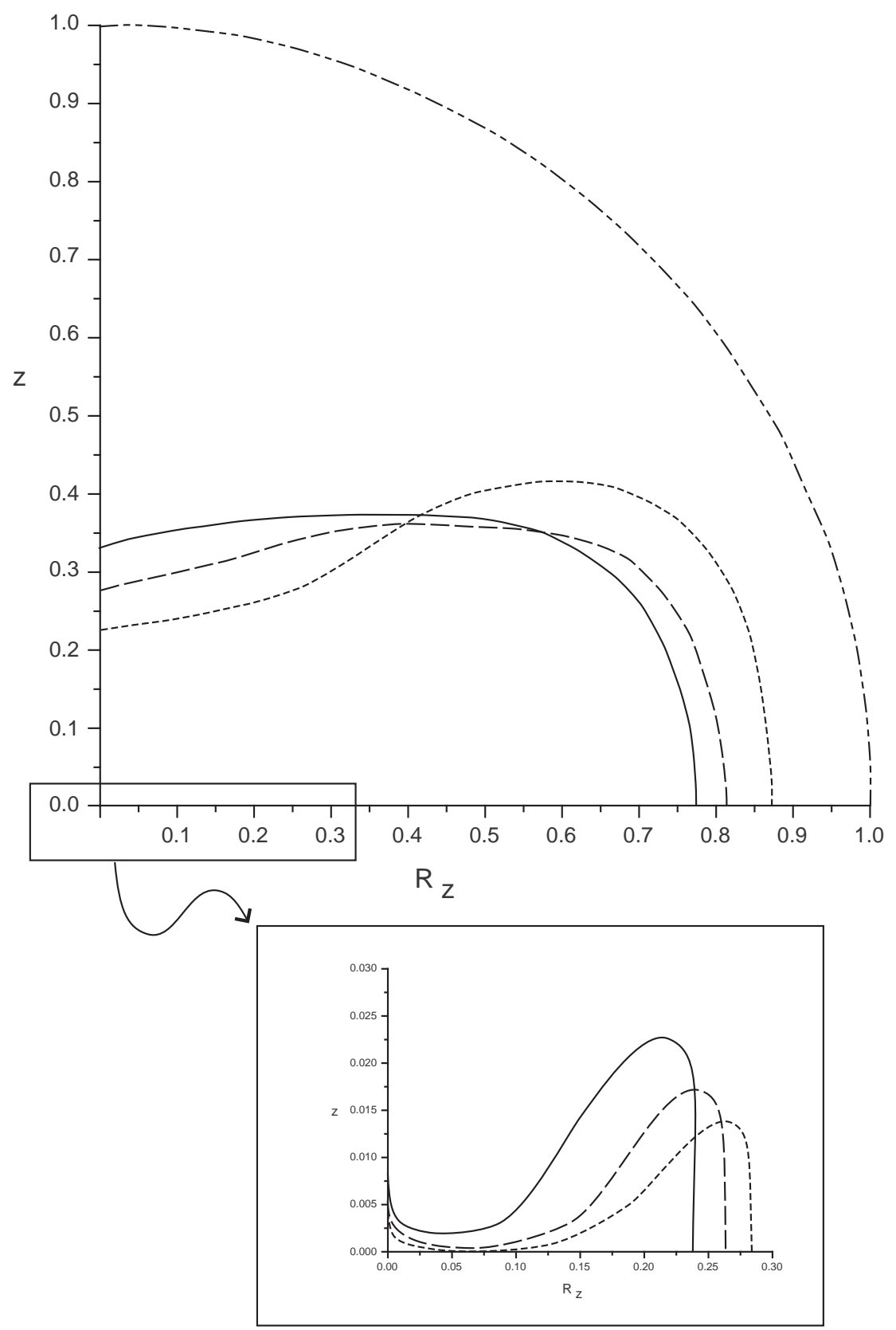

Fig. 5.- The typical iso-density contours for $\rho \approx 0.01$ (top panel), and $\rho \approx 0.1$ (bottom panel), for different values of free parameter $\tilde{B}=1$ (solid), $\tilde{B}=5$ (dash), and $\tilde{B}=10$ (dot), with $\tilde{\gamma}=10^{-5}, \tilde{\epsilon}=10^{5}$ and $\tilde{n}_{n}=1$. 